\title{
Design and Development of Biped Humanoid Robot, AMI2, for Social Interaction with Humans
}

\author{
Hyun S. Yang, Yong-Ho Seo, Yeong-Nam Chae, Il-Woong Jeong, Won-Hyung Kang and Ju-Ho Lee \\ AIM lab, EECS dept. \\ Korea Advanced Institute of Science and Technology \\ DAEJON, Republic of Korea \\ \{hsyang, yhseo, ynchae \}@paradise.kaist.ac.kr
}

\begin{abstract}
The aim of this study is to design and implement a biped humanoid robot which can interact with humans. The developed robot consists of a self-contained body, head, two arms, with a two legged (biped) mechanism. Its control hardware includes vision and speech capabilities and various control boards such as motion controllers, with a signal processing board for several types of sensors. Using the developed robot, biped locomotion study and social interaction research were concurrently carried out. The developed robot can perform various movements with its two arms, and can track an object and a face using its active vision system. It is also able to perform emotional communications with human while it is walking.
\end{abstract}

Index Terms - Humanoid Robot, Biped walking, Social interaction

\section{INTRODUCTION}

In terms of a human symbiotic situation, it is necessary for a robot to be self-contained, be fully autonomous, and for it to have reliable mobility on dynamically changing uncertain environments. For operation in human living environments, a robot with a biped walking mechanism has many advantages. Honda's ASIMO, Sony's SDR-4X and AIST's HRP-2 and the robots of the KAWADA industry are the representative robots that utilize a biped walking mechanism [1][2][3].

Research on biped-walking robots has been increasingly conducted due to these robots' greater potential mobility compared to wheeled mobile robots, despite the fact that they are relatively unstable and difficult to control in terms of posture and motion. While wheeled mobile robots can move only on continuous and regular planes, biped robots can move over stairs, irregular planes, and discontinuous planes. Their two legs can serve as shock absorbers so that any fluctuations over such terrains can be attenuated within the two legs.

In terms of symbiotic situations involving robots and humans, it is considered necessary for a robot to be sociable in terms of thinking and feeling. In this aspect, social interaction and communication that share emotions are important issues in the development of a humanoid robot. Cog and Kismet of MIT are representative examples from this point of view [4][5].

In this study, the authors have focused on building a new biped humanoid robot with a self-contained physical body, perception to a degree which allows the robot to be autonomous, and social interaction capabilities of an actual human symbiotic robot. This study builds on previous research since 1999 by the authors related to social interaction involving the author's first generation humanoid robot, AMI, coupled with biped locomotion research using a small biped robot platform in the AIM Lab [6][7][8].

The present study describes the design and the specifications of the proposed robot, the hardware system that controls the actuators and sensors, the software system that operates the robot and the biped motion control scheme. Finally, walking experiments and future plans are described

\section{DESIGN AND MEChANICAL SPECS.}

A biped humanoid robot based on the dimensions of the human body was designed and manufactured. The lower part of the robot has two legs, which have 3, 1, and 2 degrees of freedom at the pelvis, knees, and ankles, respectively. This allows the robot to lift and spread its legs, and to bend forward at the waist. This structure, which was verified by previous research to be simple and stable for biped-walking robots, makes it possible for the robot to walk as humans walk. The shape and D.O.F arrangement of the developed robot are shown in Fig. 1.

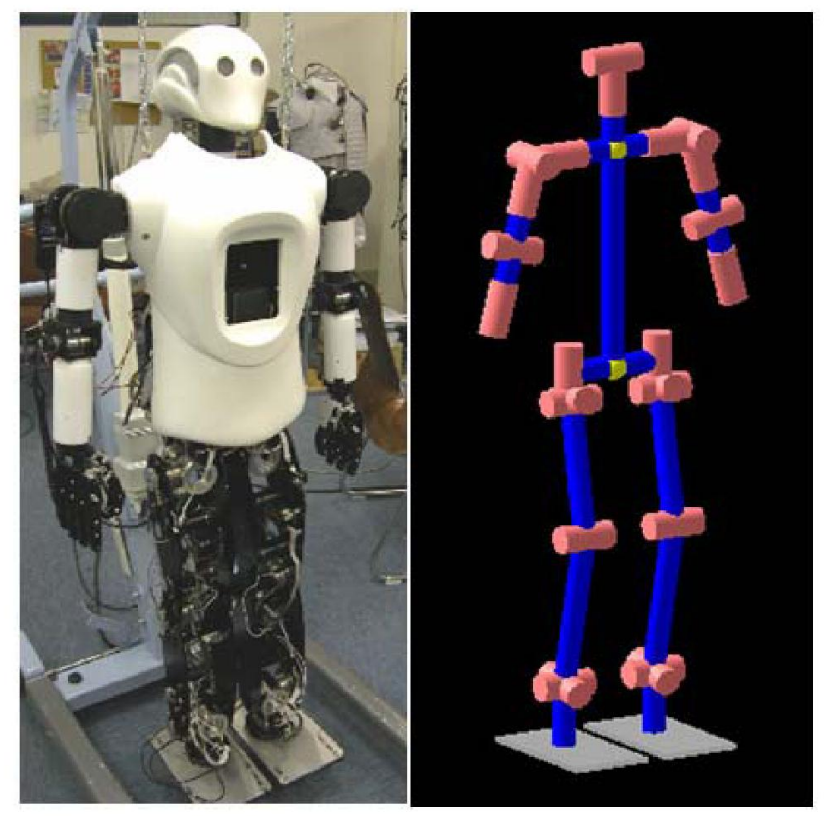

Figure 1. Appearance of AMI2 and its DOF arrangement 
Structural balance, especially for a robot with two legs, is necessary for stable motion. This type of balance involves an appropriate length of each link, the interval between the two legs, and the weight distribution of each leg. Practical and technical issues of design and manufacturing include the mechanical deployments for effective and stable actuation, a precise assembly of the linkage and actuators of the coaxial joints with two degrees of freedom, and the damping and backlash of the servomotors for stable kinematical motion during continuous activity. Most importantly, a shock and vibration absorption plate, made of rubber, is attached to the sole of the foot. For the coaxial joints with two degrees of freedom, timing belts and pulleys with bearings are installed to transmit mechanical power. Mechanical drawings of the proposed humanoid robot are shown in Fig. 2. The specification and D.O.F data for the developed robot are shown in Tables I and II, respectively.
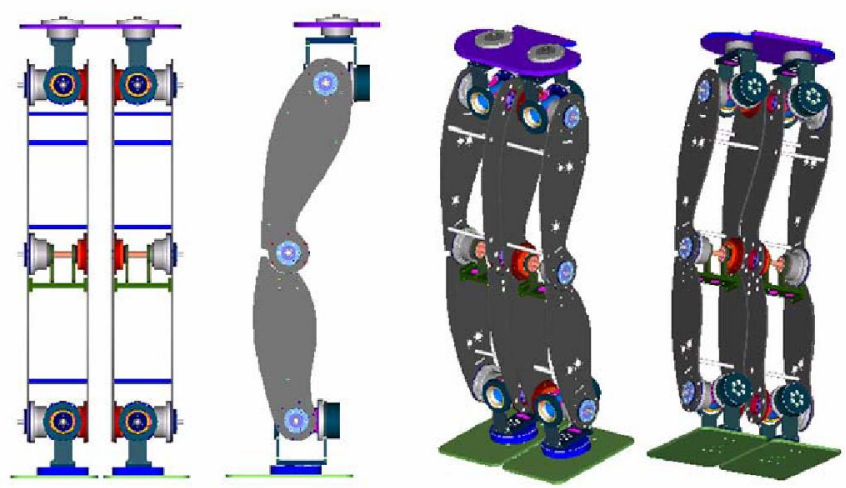

Figure 2. Mechanical Drawings of the AMI2 Biped Mechanism

TABLE I

SPECIFICATION OF AMI2

\begin{tabular}{|c|c|c|}
\hline \multicolumn{2}{|c|}{ Dimensions } & $\begin{array}{c}\text { Height: } \quad 1500 \mathrm{~mm} \\
\text { Breadth of Shoulder: } 540 \mathrm{~mm} \\
\text { Chest: } 320 \mathrm{~mm}\end{array}$ \\
\hline \multicolumn{2}{|c|}{ Weight } & $45 \mathrm{~kg}$ \\
\hline \multicolumn{2}{|c|}{ Total D.O.F } & 36 \\
\hline \multicolumn{2}{|c|}{ Walk } & $0 \sim 1 \mathrm{~km} / \mathrm{h}$ \\
\hline \multicolumn{2}{|c|}{ Grasping force } & $\sim 0.5 \mathrm{~kg} / \mathrm{hand}$ \\
\hline \multirow[t]{5}{*}{ Sensors } & Motor & Magnetic and optical encoder \\
\hline & Vision & 2 CCD camera \\
\hline & Chest & 1 axis Inclination Sensor 2EA \\
\hline & Leg & 1 axis Inclination Sensor $4 \mathrm{EA} \times 2$ \\
\hline & Foot & $\begin{array}{ll}\text { FSR sensor 4EA x2 } \\
\end{array}$ \\
\hline \multicolumn{2}{|c|}{ Actuators } & $\begin{array}{l}\text { DC servo motor } 10 \sim 90 \sim 150 \mathrm{~W} \\
\text { with Harmonic drive for arm, leg } \\
\text { RC servomotor for head, hand }\end{array}$ \\
\hline \multicolumn{2}{|c|}{ Battery } & Li-Pol Battery DC 30V,11Ah \\
\hline
\end{tabular}

TABLE II

DEGREE OF FREEDOM OF AMI

\begin{tabular}{|c|c|c|}
\hline Neck & \multicolumn{2}{|c|}{2 D.O.F } \\
\hline Arms & $\begin{array}{l}\text { Shoulder } \\
\text { Elbow } \\
\text { Wrist }\end{array}$ & $\begin{array}{l}3 \text { D.O.F } \times 2 \\
1 \text { D.O.F } \times 2 \\
1 \text { D. } . F \times 2\end{array}$ \\
\hline Hands & & 6 D.O.F $\times 2$ \\
\hline Legs & $\begin{array}{l}\text { Hip } \\
\text { Knee } \\
\text { Ankle }\end{array}$ & $\begin{array}{l}3 \text { D.O.F } \times 2 \\
1 \text { D.O.F } \times 2 \\
2 \text { D. } . F \times 2\end{array}$ \\
\hline Total & 36 D.O. & \\
\hline
\end{tabular}

New 6-DOF hands were developed for AMI2. Each finger has a simple triple-connected link structure driven by one $\mathrm{R} / \mathrm{C}$ servo motor. The hand has 6-DOFs and is actuated by six slim high-torque $\mathrm{R} / \mathrm{C}$ servos that close and open the fingers. For the thumb, 2-DOFs were used to allow for strong grasping and to widen the hands when opened. The hands of this robot are nearly same size as a human hand and weigh $420 \mathrm{~g}$ each. Their maximum payload is $0.5 \mathrm{~kg}$ each.

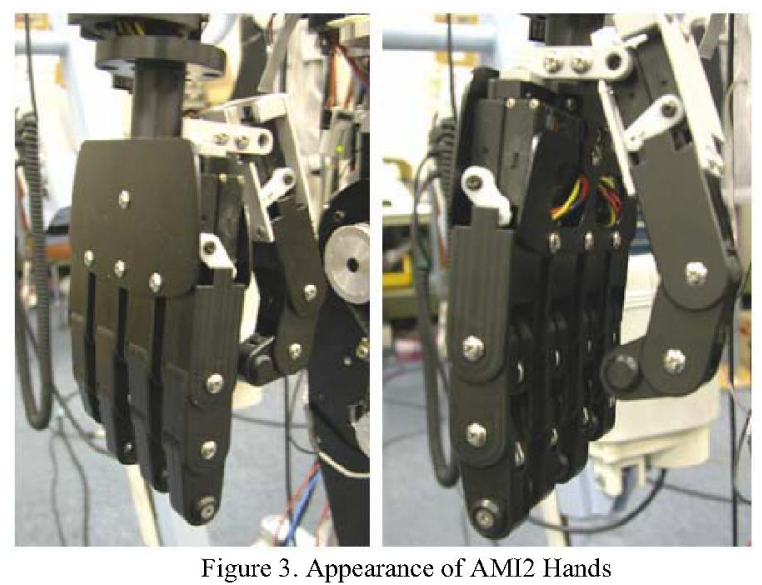

III. MOTION AND SENSOR UNITS

For the purpose of controlling the motors in the head, the arms, and the waist, a compact motion controller, compared to the other existing motion controllers, was developed. It was compact enough to be embedded into the robot body. This motion controller utilizes an ATmega162 RISC MCU, which has four PWM timers for motion control. It was designed to be able to control four DC motors concurrently. Two types of the motor driver chips, LM629 and LM18200T, were chosen from National Semiconductor and the 10A8DD module was chosen from Advanced Motion Controls. These small and high-power motor driver chips and modules were used for the arm and leg de motor controls. In particular, dual LM18200T chips incorporating a BI-amplifying method were used for the leg parts to satisfy the desired current of the motor. To control 
the two $\mathrm{R} / \mathrm{C}$ servo motors in head and six $\mathrm{R} / \mathrm{C}$ servo motors in each hand, an SMC-pro module from COMFILE technology is utilized. This was designed to be able to control eight $\mathrm{R} / \mathrm{C}$ servo motors simultaneously.

The sensor controller was designed to measure the inclination sensors and FSR sensors for posture control. The sensor boards were developed using three ATmega8535 for 24-channel A/D signal acquisitions. The microcontroller ATmega8535 has eight channels.

The high-speed RS232 interfaces of each control board were converted to USB interfaces through the use of FTDI RS232-USB conversion chips. The resulting USB interface for the robot enabled easy connection to a PC system compared to other robot systems. All motion and sensor units were connected to a USB Hub using six FTDI chips at a speed of $921600 \mathrm{BPS}$ for the motion control boards, 19200BPS for the sensor acquisition board and 9600BPS for the R/C servo control board. A hardware control structure diagram of the robot as well as the figures for both the motion controller and motor drivers are shown in Figs. 4 and 5, respectively.

To balance the robot's posture while walking, ten 1-axis inclination sensors were utilized. The position alignments of each inclination sensors are shown in Fig. 6. A total of five sets of sensors are used. A set is made up of two sensors and can measure both the $\mathrm{x}$-axis and $\mathrm{y}$-axis inclinations.

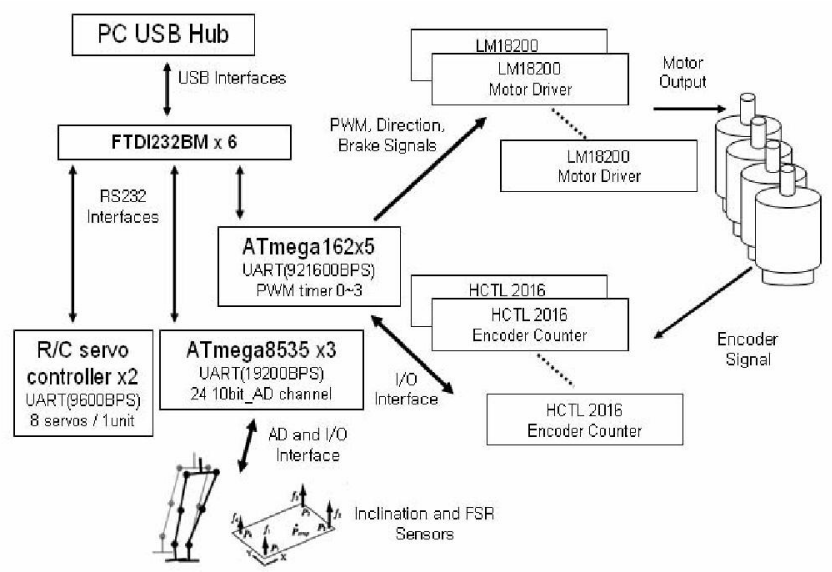

Figure 4. Hardware Control Structure Diagram
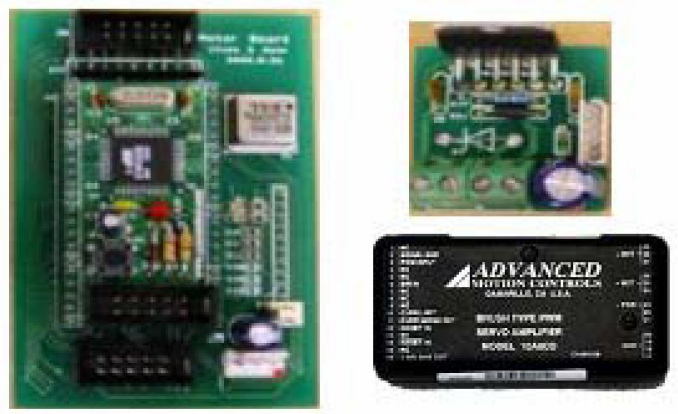

Figure 5. Motion Controller and Motor Drivers

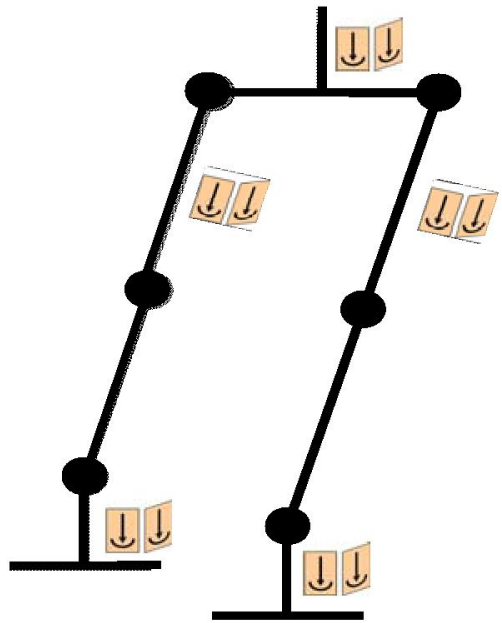

Figure 6. Position of Inclination Sensors

\section{SOFTWARE SYSTEM}

To perform high-level tasks while contending with various situations in actual, uncertain environments, a robot needs various abilities such as the capacity to detect objects using a camera in addition to the speech processing, the ability to sense an obstacle using several sensors, and the capability of manipulation and bipedal navigation.

In addition, it is necessary to integrate these software functions efficiently and reliably. Therefore, to operate the robot, unified control architecture was planned based on behavior architecture. The designed architecture performs complex tasks successfully using a set of coordinated behaviors. A high-level task is driven from a motivation system. The motivation system activates set of coordinated behaviors. Finally, an expression system activates multimodal human interfaces such as a voice, gestures and 3D facial expressions.

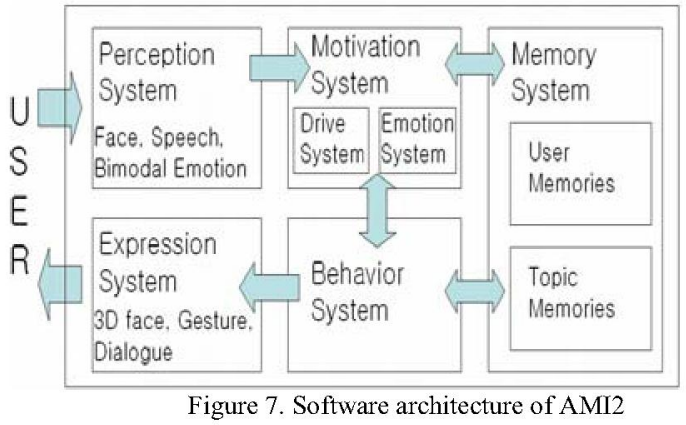

A. Perception System

1) Face Detection and Recognition: The face detection system uses a bottom-up, feature-based approach. The system searches an input image for a set of facial features, and groups regions into face candidates based on the geometric relationship of the facial features. Finally, the system determines whether the candidate region is a face by locating eyes in the eye region of a candidate's face. The detected image is sent to the face recognizer and to the emotion recognizer. Face recognizer determines the user's identity from face database. 
2) Bimodal Emotion Recognition: Emotion is estimated through facial expression and speech; they are then integrated to enable bimodal emotion recognition. For emotion recognition through facial expression, the captured image was normalized. The following two features are then extracted, which are based on Ekman's facial expression features [9]: The first is a facial image of the lips, brow and forehead, and the second is an edge image of the lips, brow and forehead. Following this, for emotion recognition through speech, a recognition method similar to the one used in the life-like communication agents MUSE and MIC [10] was adopted. The proposed system extracts two features: a phonetic feature and a prosodic feature. A neural network was used to train each feature vector. For bimodal emotion recognition, decision logic was used to integrate the two training results. For more detailed information on implementation, see reference paper $[8]$.

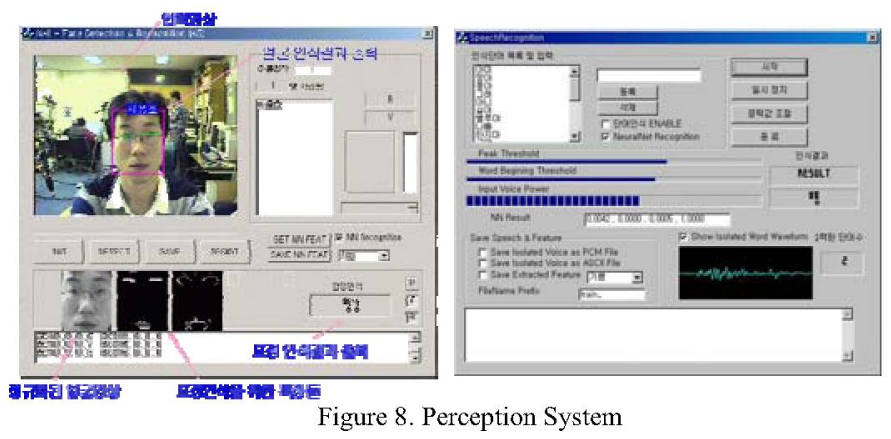

\section{B. Motivation System}

The motivation system defines the robot's nature by defining its "needs" and influencing how and when it acts to satisfy them. The nature of the proposed humanoid robot is to socially interact with humans and ultimately to ingratiate itself with them. The motivation system consists of two related subsystems, one that implements drives and a second that implements emotions. Each subsystem serves as a regulatory function for the robot to maintain its "well-being"

In previous research, three basic drives were defined for a robot's affective communication with humans [8][12]. In the drive system for AMI2, four basic drives were defined for the robot's objectives as they related to social interaction with a human: a drive to obey a human's commands; a drive to interact with a human; a drive to ingratiate itself with humans and a drive to maintain its own well-being.

The first drive motivates AMI2 to perform a number of pre-defined services according to a human's commands. The second drive activates the robot to approach and greet humans. The second drive prompts the robot to try to improve a human's feelings. When the robot interacts with humans, it tries to ingratiate itself while considering the human's emotional state. The third drive is related to robot's maintenance of its own well-being. When the robot's sensors tell it that extreme anger or sadness is appropriate, or when its battery is too low, it stops interacting with humans.

\section{BIPED MOTION CONTROL}

Fig. 9 shows a block diagram of the control for walking. First, an operator sets several walking parameters, such as the step length, beginning and ending ankle angle, step period, and double support phase ratio. In the second step, the user constructs a motion list which is made up of a motion sequence. In the third step, the system generates a walking pattern based on the motion type and walking parameters. A walking trajectory is used that is generated by a constrained third-order spline interpolation. This walking trajectory provides the largest stability margin to the biped walking robot [11].

The walking pattern is a modified trunk roll/pitch controller, a thigh pitch controller and a sole roll/pitch controller. In the forth step, the joint angles of each interpolation point are derived by inverse kinematics. From each joint angle of each interpolation point, the system generates the joint angle pattern during the fifth stage. Finally, the system sends the joint angle data to all joint motor controllers. The motor controller operates the motor using the PD control scheme.

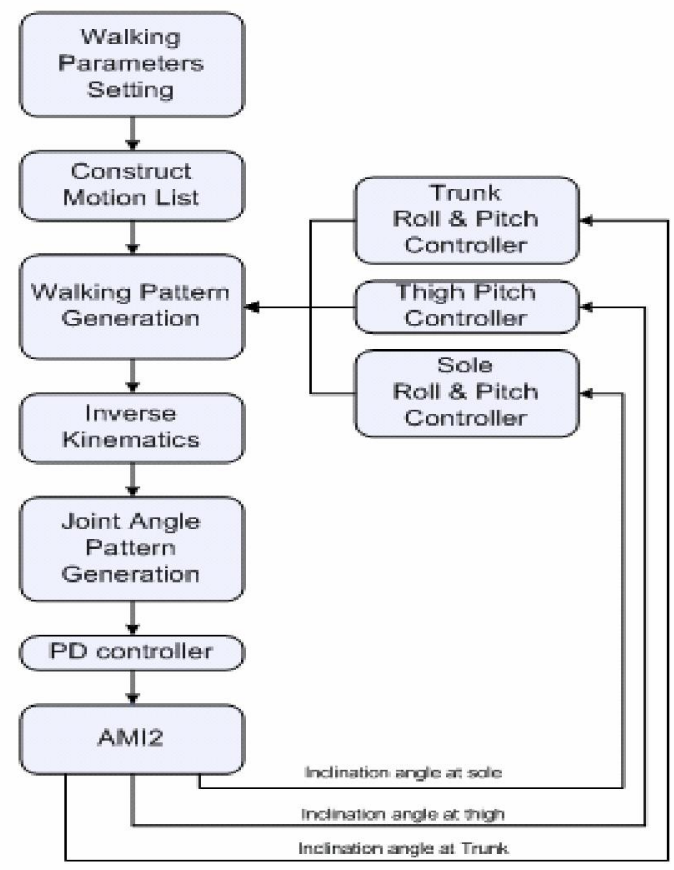

Figure 9. Block diagram of the walking control

For the generated walking trajectory, ZMP is in each supporting leg's sole. If the biped robot walks using the generated walking trajectory, the robot will not collapse. However, when the biped robot walks based on the walking trajectory, it will fall. This indicates that the robot's walking posture is different from its trajectory posture. This disparity is caused by backlash of the joint and the biped robot's weight. To adjust the robot's posture to its trajectory, ten 1axis inclination sensors were used. Using 1-axis inclination sensors, it was possible to reduce the biped robot's unit cost of production and save power. Saving power allows more 
mobility. Table III shows a cost and power consumption comparison between an inclination sensor and a force-torque sensor.

TABLE III

COST \& POWER CONSUMPTION OF EACH SENSOR

\begin{tabular}{|c|c|c|}
\hline & $\begin{array}{c}6 \text {-axis force-torque } \\
\text { sensor }\end{array}$ & $\begin{array}{c}1 \text {-axis inclination } \\
\text { sensor }\end{array}$ \\
\hline Cost & $10,000 \$ \times 2=20,000 \$$ & $25 \$ \times 10=250 \$$ \\
\hline $\begin{array}{c}\text { Power } \\
\text { Consumption }\end{array}$ & $50 \mathrm{~W} \times 2=100 \mathrm{~W}$ & $5 \mathrm{~mW} \times 10=50 \mathrm{~mW}$ \\
\hline
\end{tabular}

The disparity appears to cause the biped robot's posture to lean. This lean is a critical property that disturbs stable walking. The lean was analyzed and decomposed to the roll and pitch directions, as actuators that sustain the robot's weight are only in the roll and pitch directions. In general, the lean in the roll direction has a more detrimental influence on stable walking compared to the lean in the pitch direction. To measure the inclination of the roll and pitch direction, sets of inclination sensors were installed orthogonally. A pair of sensors was installed in the chest. Each leg has two pairs of sensors, one pair in the thigh and the other in on sole. Using these sensors, it was possible to measure roll and pitch inclination of the trunk, thigh, and sole.

During the initial marking time of walking, the robot compensates for its lean using the inclination sensors. The walking trajectory is then regenerated based on the compensation result. Fig. 10 shows the process of compensation. The grey posture is the desired posture and the black posture is the leaning posture. Except for the first stage, compensation spreads from the supporting leg to the swing leg through the trunk. As the supporting sole makes contact with the ground, only the supporting sole has the correct posture.

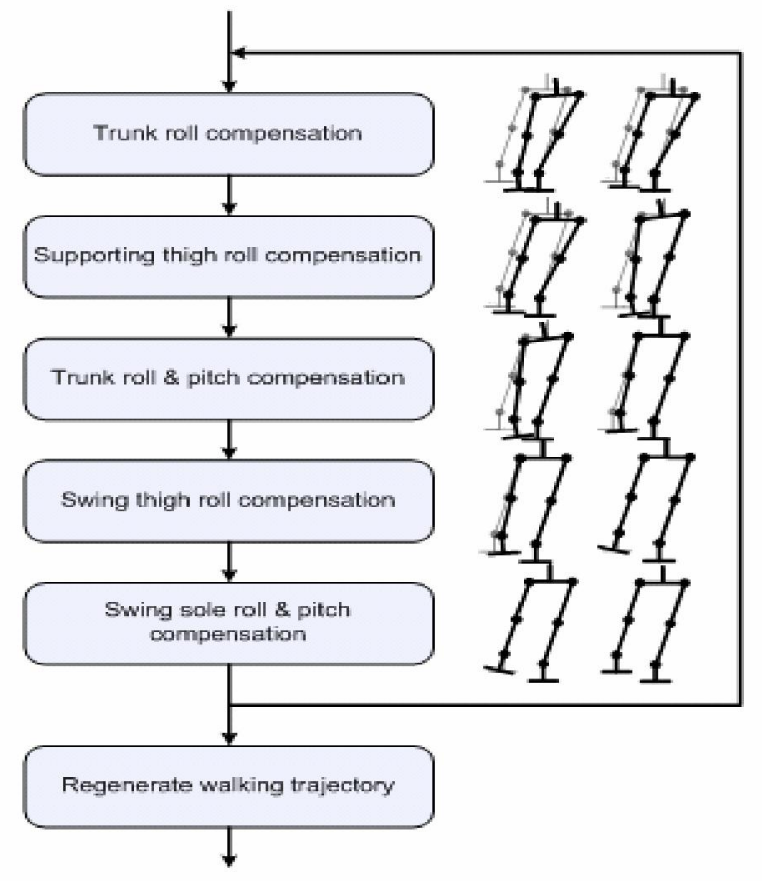

Figure 10. Compensation process
Each stage compensates for the robot's posture using the algorithm in Fig. 11, in which $\mathrm{u}$ is the joint angle, $\mathrm{c}$ is the compensation angle, $\mathrm{d}$ is the desired angle, $\mathrm{o}$ is the observed angle, te is the tolerance error, and $\mathrm{k}$ is a constant value identified by the experiment. This algorithm shows that the biped robot adjusts each joint until the observed angle arrives at the desired angle within a tolerance error. During the initial marking time of walking, compensation was performed at four instances.

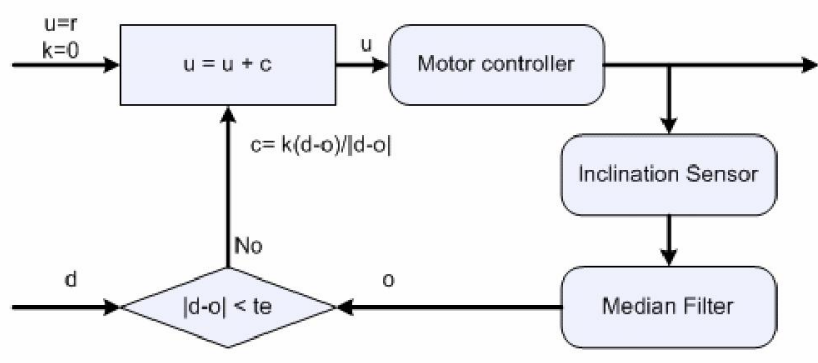

Figure 11. Compensation algorithm

To increase stability, four FSR sensors were installed under each sole. From these FSR sensors, it was possible to measure the ZMP (Zero Moment Point) via the equation shown in Fig. 12. Fig. 13 shows the proposed biped robot's yaxis ZMP.

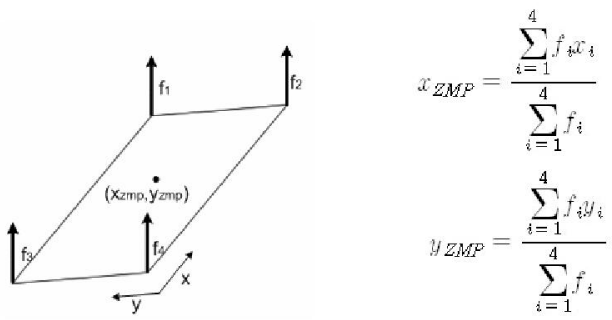

Figure 12. ZMP from the FSR sensors

\section{EXPERIMENT}

A number of basic walking experiments were conducted with the proposed robot in an effort to verify its ability. Fig. 14 shows the sequence of walking experiments. These figures illustrate the different steps in biped walking. They show the waist swinging phase, and the start, progress, and end of the walk.

We had also some basic experiments with our robot to verify the ability of its social interaction. Some examples of these experiments are following. Conversations with robot such as "say hi", "let's shake hands", "good job" and "not good" show examples of basic robot-human interaction. At this time, Operator can see the emotional interface in the middle of chest of robot for emotional communication. The unified control architecture which manages speech, vision, manipulation with two arms was very successful. 

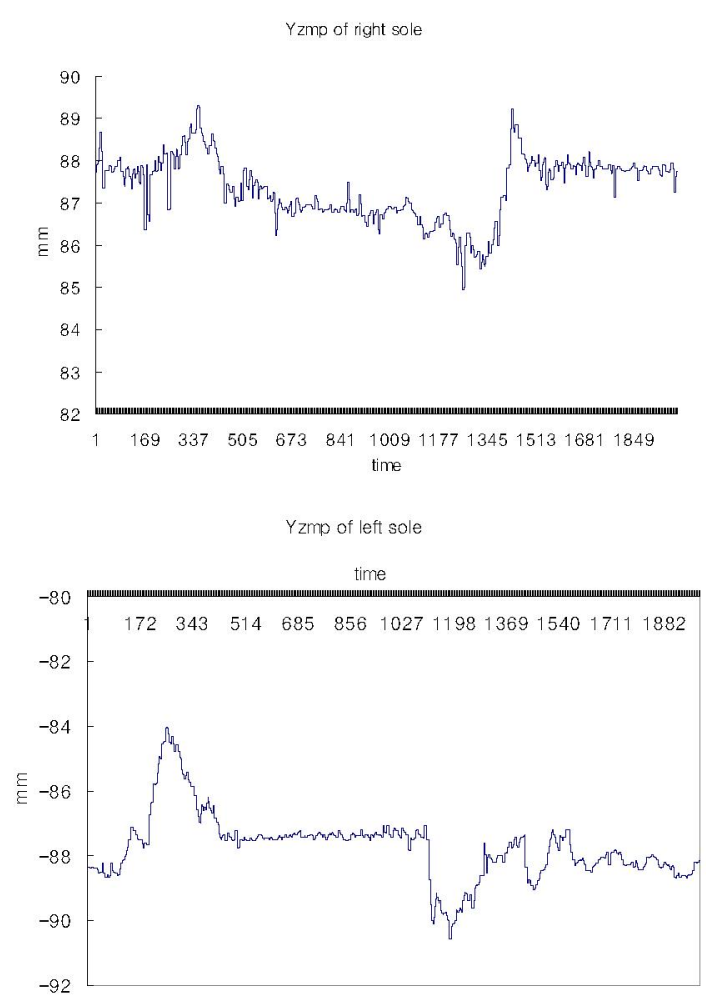

Figure 13. The y-axis ZMP of each sole

\section{CONCLUSION}

This paper presented the design concept, hardware system and software system of the biped humanoid robot AMI2, as well as a description of its biped walking. The developed robot has a human-like head, two arms and two legs. The robot has several sensors that obtain postural data and obtain contact force data. In addition, the robot has a software system based on the unified control architecture. The biped walking capability of the robot was proven through a walking experiment.

The walking patterns were generated through the ZMP method; as a result, the robot is able to walk stably. A realtime posture control method, utilizing ten 1-axis inclination sensors installed in the robot's trunk and each joint of its leg is proposed. The median filter is used to eliminate noise from the sensor signal, and the algorithm is implemented to stably maintain the trunk horizontally. The robot can control its posture steadily while it is walking as a result.

To make its walk more stable, additional meaningful measures regarding the landing motion from the FSR sensors are needed. Thus, the authors plan to investigate a method that extracts these additional meaningful measures. In addition, a plan has been made to design a control scheme based on the FSR sensors. Moreover, a hybrid control scheme that uses both inclination sensors and FSR sensors is under investigation.

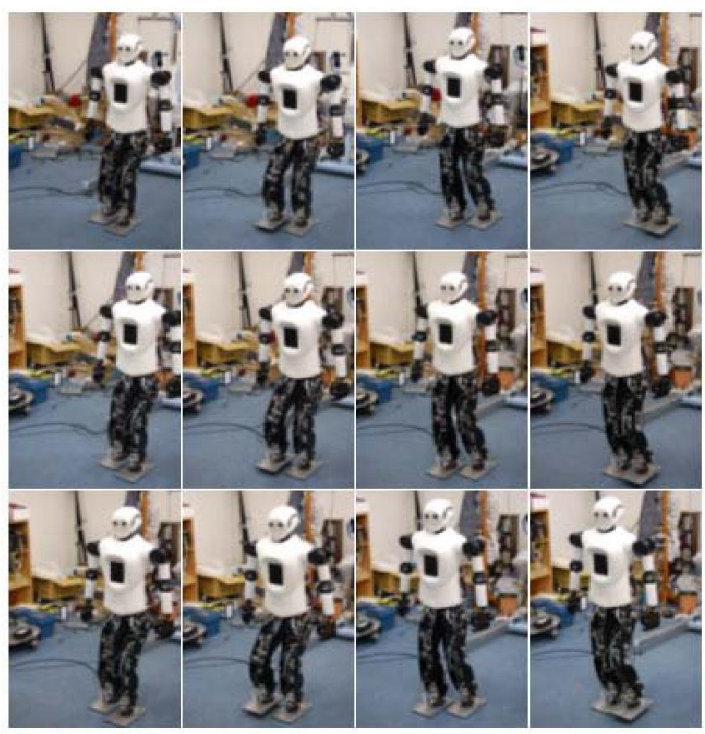

Figure 14. Walking Experiment

ACKNOWLEDGMENT

This research was partially supported by the Korea Ministry of Commerce, Industry and Energy through the Brain Science Research Project and the Korea Ministry of Science and Technology through AITRC, as well as by the Information Technology Research Center (ITRC) Program of the Ministry of Information and Communication (MIC) in Korea.

\section{REFERENCES}

[1] http://world.honda.com/ASIMO/

[2] http:/www.sony.net/SonyInfo/News/Press/200203/02-0319E/.

[3] Noriyuki Kanehira, Toshikazu Kawasaki, Shigehiko Ohta, et.al, "Design and Experiments of Advanced Leg Module (HRP-2L) for Humanoid Robot (HRP-2) Development", Proceedings of IEEE International Conference on Intelligent Robots and Systems, pp. 2455-2460, 2002.

[4] R. A. Brooks, C. Breazeal, R. Irie, C. C. Kemp, M. Marjanovic, B. Scassellati, M. Williamson, "Alternate Essences of Intelligence", American Association for Artificial Intelligence (AAAI), 1998.

[5] Reazeal, C. and Scassellati, B., "A Context-Dependent Attention System for a Social Robot", In Proceedints of the Sixteenth International Joint Conference on Artificial Intelligence (IJCAI'99), pp.1146-1151, 1999.

[6] Yong-Ho Seo, Ho-Yeon Choi, Il-Woong Jeong, and Hyun S. Yang, "Design and Development of Humanoid Robot AMI for Emotional Communication and Intelligent Housework," Proceedings of IEEE-RAS HUMANDOIS 2003, pp.42 (2003)

[7] T.M. Kim, Yong-Ho Seo, T.W. Kim, N. Raess, Hyun S. Yang, "Development of the Small Biped-Walking Robot Platform for the Biped Locomotion Research", HUMANOIDS 2003.

[8] Jung, H., Seo, Y., Ryoo, M.S., Yang, H.S., "Affective Communication System with Multimodality for the Humanoid Robot AMI", HUMANOIDS 2004

[9] Ekman, P., Friesen, W.V.: Facial Action Coding System: Investigator's Guide. Consulting Psychologists Press, Palo Alto, CA (1978)

[10]Tosa, N., Nakatsu, R.: Life-like Communication Agent - Emotion Sensing Character "MIC" \& Feeling Session Character "MUSE.", ICMCS (1996)

[11]Qiang Huang, Kazuhito Yokoi, Shuuji Kajita, etc, "Planning Walking Patterns for a Biped Robot", IEEE TRANSACTIONS ON ROBOTICS AND AUTOMATION, VOL. 17, NO. 3, JUNE 2001

[12]M. S. Ryoo, Yong-ho Seo, Hye-Won Jung, and H. S. Yang, "Affective Dialogue Communication System with Emotional Memories for Humanoid Robots", Springer LNCS3784, Affective Computing and Intelligent Interaction 2005 (ACII2005) 